\title{
Transposição do conhecimento científico no ensino de Matemática para cegos
}

\author{
Transposition of scientific knowledge in teaching Mathematics to the blind \\ Transposición del conocimiento científico en la enseñanza de las Matemáticas a ciegos
}

Recebido: 18/11/2020 | Revisado: 29/11/2020 | Aceito: 24/03/2021 | Publicado: 25/03/2021

\author{
Guilherme Santos Simões \\ ORCID: https://orcid.org/0000-0001-9050-0401 \\ Universidade Cruzeiro do Sul, Brasil \\ E-mail: gui.quimico.unifal@gmail.com \\ Rafael Soares Silva \\ ORCID: https://orcid.org/0000-0001-9994-6653 \\ Universidade Cruzeiro do Sul, Brasil \\ E-mail: doc.rafaelsoares@gmail.com \\ Carmem Lúcia Costa Amaral \\ ORCID: https://orcid.org/0000-0002-6495-153X \\ Universidade Cruzeiro do Sul, Brasil \\ E-mail: carmem.amaral@cruzeirodosul.edu.br
}

\begin{abstract}
Resumo
Esse estudo teve como objetivo investigar os desafios e potencialidades na transposição da linguagem científica no ensino de matemática para alunos com deficiência visual. A pesquisa possui uma abordagem qualitativa e como forma de apropriação e percepção de temas elementares para a efetivação do estudo realizou-se uma revisão de literatura. Na ausência da visão meios alternativos devem ser utilizados a fim de favorecer a familiarização com as ciências e a construção do conhecimento de maneira semelhante aos demais alunos. Constatou-se que existe uma dificuldade na transposição do conhecimento científico para o conhecimento escolar devido, sobretudo, a inexistência de manuais específicos de como realizá-las de maneira satisfatória, devido a especificidade da linguagem científica. Soma-se a esta ausência a falta de preparo dos professores, das escolas, e a carência de materiais pedagógicos adaptados como desafios a serem superados a fim de transpor a linguagem científica para as pessoas com diferentes necessidades de aprendizagem
\end{abstract}

Palavras-chave: Divulgação científica; Ensino de Matemática; Deficiência visual.

\begin{abstract}
This study aimed to investigate the challenges and potentialities in the transposition of scientific language in the teaching of mathematics for students with visual impairment. The research has a qualitative approach and as a form of appropriation and perception of elementary themes for the effectiveness of the study, a literature review was carried out. In the absence of a vision, alternative means should be used in order to promote familiarization with the sciences and the construction of knowledge in a similar way to other students. It was found that there is a difficulty in transposing scientific knowledge to school knowledge due, above all, to the lack of specific manuals on how to perform them satisfactorily, due to the specificity of scientific language. Adding to this absence is the lack of preparation of teachers, schools, and the lack of teaching materials adapted as challenges to be overcome in order to transpose the scientific language to people with different learning needs.
\end{abstract}

Keywords: Scientific dissemination; Teaching Mathematics; Visual impairment.

\section{Resumen}

Este estudio tuvo como objetivo investigar los desafíos y potencialidades en la transposición del lenguaje científico en la enseñanza de las matemáticas para estudiantes con discapacidad visual. La investigación tiene un enfoque cualitativo y como forma de apropiación y percepción de temas elementales para la efectividad del estudio se realizó una revisión de la literatura. En ausencia de una visión, se deben utilizar medios alternativos para promover la familiarización con las ciencias y la construcción del conocimiento de manera similar a otros estudiantes. Se encontró que existe una dificultad en la transposición del conocimiento científico al conocimiento escolar debido, sobre todo, a la falta de manuales específicos sobre cómo realizarlos de manera satisfactoria, debido a la especificidad del lenguaje científico. A esta ausencia se suma la falta de preparación de los docentes, las escuelas y la falta de materiales didácticos adaptados como desafíos a superar para trasponer el lenguaje científico a personas con diferentes necesidades de aprendizaje.

Palabras clave: Divulgación científica; Enseñanza de las matemáticas; Discapacidad visual. 


\section{Introdução}

Verifica-se a cada ano um aumento do número de matrículas na Educação Especial Básica, a quantidade de matrículas de estudantes com deficiência visual passou de 24.185 em 2003 para 75.433 em 2015 (INEP, 2015). No ano de 2019, foram registradas 47,9 milhões de matrículas nas 180,6 mil escolas de Educação Básica no Brasil. Desse total, 1,3 milhões corresponde as matrículas da Educação Especial (INEP, 2019).

A Tabela 1 apresenta o número de matrículas de alunos que apresentam cegueira e baixa visão matriculados em classes comuns ${ }^{1}$.

Tabela 1: Número de matrículas de alunos que apresentam cegueira e baixa visão em classes comuns por região brasileira.

\begin{tabular}{l|c|c}
\hline Regiões do País & Cegueira & Baixa Visão \\
\hline Norte & 804 & 9.930 \\
\hline Nordeste & 1.908 & 28.114 \\
\hline Centro-Oeste & 543 & 4746 \\
\hline Sudeste & 2.212 & 20.926 \\
\hline Sul & 785 & 10.123 \\
\hline Total & 6.252 & 73.839 \\
\hline
\end{tabular}

Fonte: INEP (2020).

Além das escolas regulares existem aquelas que são exclusivamente especializadas em receber alunos com NEE. A Tabela 2 mostra o número de matrículas de alunos cegos e com baixa visão nessas escolas por região do Brasil.

Tabela 2: Matrículas de alunos com deficiência visual, em Escolas Exclusivamente Especializadas e/ou em Classes Exclusivas $^{2}$ de Ensino Regular e/ou EJA.

\begin{tabular}{l|c|c}
\hline Regiões do País & Cegueira & Baixa Visão \\
\hline Norte & 48 & 221 \\
\hline Nordeste & 123 & 457 \\
\hline Centro-Oeste & 93 & 315 \\
\hline Sudeste & 686 & 1.379 \\
\hline Sul & 275 & 1.117 \\
\hline Brasil & 1.225 & 3.489 \\
\hline
\end{tabular}

Fonte: INEP (2020).

Para atender a demanda crescente do número de estudantes com deficiência visual é necessário que o sistema se adeque e proporcione a inclusão do aluno com deficiência. As instituições mostram-se importantes, como aponta Mantoan (2003):

\footnotetext{
1 Classes comuns: Inclui matrículas de alunos com algum tipo de deficiência, transtorno global do desenvolvimento e altas habilidades/superdotação de Ensino Regular e/ou EJA.

2 Classes Exclusivas - Inclui matrículas de alunos com algum tipo de deficiência, transtorno global do desenvolvimento e altas habilidades/superdotação em Escolas Exclusivamente Especializadas e/ou em Classes Exclusivas de Ensino Regular e/ou EJA.
} 
As escolas inclusivas propõem um modo diferenciado de organização do sistema educacional, consideram as necessidades de todos os estudantes e são estruturadas em função dessas necesidades. Por tudo isso, a inclusão implica uma mudança de perspectiva educacional, pois não atinge apenas estudantes com deficiência e os que apresentam dificuldades de aprender, mas todos os demais, para que se obtenha sucesso na corrente educativa geral (Mantoan, 2003, p. 16).

De acordo com Vilela Ribeiro e Benite (2013) uma das barreiras evidenciadas refere-se a falta de preparo dos professores e das escolas em transpor a linguagem científica para as pessoas com diferentes necessidades de aprendizagem. Esta dificuldade na transposição do conhecimento científico para o conhecimento escolar se deve, segundo Villani e Nascimento (2003), a inexistência de manuais específicos de como realizá-las de maneira satisfatória, devido a especificidade da linguagem científica.

Em relação à Deficiência Visual Vigotski (1997) estudioso da temática Defectologia reforçava que é inegável que as crianças cegas apresentam algumas limitações de vivência, o que implica diretamente no processo de desenvolvimento cognitivo e na aprendizagem escolar. O sucesso ou insucesso dessas crianças, socialmente e na escola, não está atrelado diretamente à cegueira, mas, sim, aos limites que a classes sociais e a escola delimitam. A falta de recursos provoca a limitação social do deficiente visual, prejudicando o desenvolvimento das funções psicológicas superiores (Silva,2020)

Dentre estas especificidades está a necessidades de compreensão de leis, teorias, conceitos, princípios e estruturas que compõe a linguagem científica (Vilela Ribeiro; Benite, 2013), Silva e Amaral (2020), salientam que o conhecimento matemático e científico das ciências naturais é instrumento para que o sujeito se coloque na posição ativa, capaz de tomar decisões, com a finalidade de contribuir para o desenvolvimento da sociedade. Para isso, esses conhecimentos precisam estar ao alcance de todos, de forma que seu ensino seja democratizado.

Em relação às pessoas cegas ou com deficiência visual necessitam de adaptação em Braille, equipamentos para produzir materiais em Braille e acesso a serviços de biblioteca com livros acessíveis no formato Braille ou audiobooks, além de leitores de tela e equipamento de ampliação (Silva, 2020).A pouca atenção dada à educação inclusiva durante a formação do professor e, em particular, à educação para deficientes visuais contribui significativamente para uma diminuição da produção de materiais didáticos e atividades para alunos com deficiência visual (Silva \& Amaral, 2020)

Além destas particularidades existem outros desafios a serem enfrentados. Os desafios na transposição do conhecimento matemático é abordado por Fernandes e Healy (2007), em no "Ensaio sobre a inclusão na educação matemática" as autoras evidenciam a carência de materiais necessários ao estudo de matemática, bem como a necessidade de formação apropriada dos professores uma vez que estes não se sentem preparados para atuarem em salas de aulas inclusivas.

Sabemos que a visão é o canal mais importante de comunicação do ser humano com o meio externo, pois permite captar registros próximos e distantes e, a partir desse sentido, primordialmente, o sistema nervoso central organiza as informações advindas dos outros sentidos. (GIL, 2000). Desse modo na ausência da visão meios alternativos devem ser utilizados a fim de favorecer a familiarização com as ciências e a construção do conhecimento de maneira semelhante aos demais alunos.

Sobre esta relação Almeida (1931) discorre que a divulgação científica promove a difusão das ciências, a confiança nos métodos científicos além de informar a população sobre os benefícios que a ciência pode gerar. Considerando o contexto exposto anteriormente, destaca-se a necessidade de verificar como a divulgação científica ocorre no ensino de matemática para alunos com deficiência visual, uma vez que o conhecimento científico atua como mitigador das barreiras que ocasionam a exclusão social.

Assim sendo neste artigo, analisamos os desafios e potencialidades na transposição da linguagem científica no ensino de matemática para alunos com deficiência visual. 


\section{Metodologia}

De acordo com Freire (1996, p.32) o ensino e a pesquisa são indissociáveis. A pesquisa propicia conhecer o que ainda é oculto, ela é o caminho que proporciona a comunicação e o anuncio do que antes era incompreendido. A pesquisa qualitativa possui um forte caráter descritivo, sendo, de acordo com Manning (1979, p.668), uma característica fundamental neste tipo de estudo.

Devido ao uso da revisão de literatura como forma de apropriação e percepção de temas elementares para a efetivação do estudo, essa pesquisa terá uma abordagem qualitativa. Nessa perspectiva adotar-se-á o raciocínio hipotético-dedutivo na análise e interpretação de dados.

De acordo com Moreira (2004) aplica-se a pesquisa básica na busca de respostas a perguntas sobre o ensino, aprendizagem, currículo, contexto educativo e formação permanente do professor de ciências. Por estes motivos a pesquisa possuirá natureza aplicada.

A pesquisa exploratória possui como objetivo principal o aprimoramento de ideias, descoberta de intuições, a possibilidade de explorar diferentes aspectos relativos ao fato estudado e favorecer a familiarização com o problema (Gil,2002).

A pesquisa proposta classifica-se como exploratória visto que se utilizou de fontes bibliográficas e descritivas com intuito de propiciar a familiarização com os objetivos da investigação. Como instrumento de coleta de dados optou-se pelo levantamento bibliográfico, a fim de se obter uma melhor apreciação do conteúdo foco do nosso estudo.

A princípio a busca de artigos científicos que correspondessem aos critérios de inclusão se deu no Portal Periódicos da Capes com os descritores deficiência visual e ensino de matemática. Os descritores definidos foram selecionados com intuito de ampliar o campo analítico inicial. Considerou-se os termos presentes apenas nos títulos e palavras-chave em todos os artigos dos últimos 10 anos.

Com os artigos selecionados conforme os critérios de inclusão previamente definidos, efetuou-se os seguintes passos: leitura exploratória; leitura seletiva e escolha do material que se adequam aos objetivos e tema deste estudo. Estas etapas foram seguidas com intuito de construir o corpus de nossa pesquisa e evidenciar o panorama do ensino de matemática para alunos deficientes visuais

\section{Resultados e Discussão}

Este estudo teve o propósito de verificar como sobrevém o ensino de matemática para alunos com deficiência visual. Uma vez que se verifica um aumento no número de educandos com necessidades especiais de aprendizagem no ensino público regular. Faz-se necessário investigar como ocorre a transposição do conhecimento científico matemático para o conhecimento escolar.

Com objetivo de atingirmos nossas finalidades e para uma melhor percepção do tema em estudo elaborou-se o Quadro 01 com as produções que surgiram desta investigação. 
Quadro 1: Produções proveniente do Banco de Teses CAPES no período de 2010-2020.

\begin{tabular}{|l|c|l|}
\hline ANO & AUTOR(ES) & \multicolumn{1}{|c|}{ ARTIGOS } \\
\hline $\mathbf{( 2 0 1 0 )}$ & Shimazaki, Silva, Viginheski & $\begin{array}{l}\text { Ensino de conceitos matemáticos para estudante com deficiência visual } \\
\text { em situação de inclusão. }\end{array}$ \\
\hline $\mathbf{( 2 0 1 5 )}$ & Shimazaki, Silva, Viginheski & $\begin{array}{l}\text { O ensino de matemática e a diversidade: o caso de uma estudante com } \\
\text { deficiência visual. }\end{array}$ \\
\hline $\mathbf{( 2 0 1 6 )}$ & Pasquarelli, Manrique & $\begin{array}{l}\text { A inclusão de estudantes com deficiência visual no ensino e } \\
\text { aprendizagem de estatística: medidas de tendência central. }\end{array}$ \\
\hline $\mathbf{( 2 0 1 8 )}$ & Ferreira, Corrêa e Boron & O Ensino da Matemática para Portadores de Deficiência visual. \\
\hline $\mathbf{( 2 0 1 9 )}$ & Cintra, Beirigo & $\begin{array}{l}\text { Deficiência Visual e Educação Matemática: estudo dos artigos } \\
\text { publicados nos anais dos Encontros Nacionais de Educação Matemática. }\end{array}$ \\
\hline
\end{tabular}

Fonte: Dados da pesquisa.

No artigo "O Ensino de Matemática e a diversidade: o caso de uma estudante com deficiência visual" e "Ensino de conceitos matemáticos para estudante com deficiência visual em situação de inclusão" a s autoras Shimazaki, Silva, Viginheski (2015) discutem sobre uma proposta de ensino que promova o desenvolvimento do pensamento algébrico e favoreça a inclusão, pautados em estudos bibliográficos, pesquisa de campo e intervenção pedagógica discorrem sobre a importância do uso de diferentes recursos metodológicos para o ensino de matemática.

As autoras abordam o conceito de produtos notáveis a partir da utilização do jogo "Prenda o rei". De acordo com Shimazaki, Silva, Viginheski (2015):

O jogo Prenda o Rei, de origem desconhecida, executado em duplas, foi utilizado para motivar os estudantes para a aprendizagem, explorar os conceitos de perímetro e área e, posteriormente, o desenvolvimento dos produtos notáveis de quadrado da soma, quadrado da diferença e produto da soma pela diferença. Utilizam-se, do tabuleiro de xadrez, os dois reis e as fichas coloridas para serem colocadas no tabuleiro durante o jogo. Cada jogador, em sua vez, movimenta o seu rei (o mesmo movimento do xadrez) e coloca uma ficha colorida sobre uma casa, com a finalidade de prender o rei adversário. Nenhum dos reis pode ocupar uma casa coberta pela ficha colorida. Vence o primeiro que prender o rei adversário.

Durante a construção do conhecimento científico as professoras utilizam-se do lúdico como forma de despertar o interesse e curiosidade dos alunos. Segundo as autoras o ensino de matemática pautado em práticas pedagógicas tradicionais reduz o ensino da disciplina à apresentação de conceitos previamente elaborados, inviabilizando a construção do conhecimento científico. As práticas hegemônicas são apontadas como barreiras na edificação do conhecimento e desenvolvimento de práticas que atendam a diversidade.

Somam-se a estes obstáculos a necessidade de tempo na construção de significados de conceitos científicos tais como aqueles que envolvem os conceitos estatísticos. Esta adversidade é abordada por Pasquarelli, e Manrique (2016) no artigo "A inclusão de estudantes com deficiência visual no ensino e aprendizagem de estatística: medidas de tendência central" onde as autoras abordam os conceitos de média, moda e mediana através do uso da tecnologia assistiva. Constata-se, a partir da leitura, que a tecnologia assistiva favorece o processo de ensino e aprendizagem por promover a autonomia dos estudantes cegos. Além disto o ensino e aprendizagem de estatística baseado na utilização das diferentes tecnologias vão de encontro as diretrizes dos Parâmetros Curriculares Nacionais: 
A formação do aluno deve ter como alvo principal e aquisição de conhecimentos básicos, [...] utilizar as diferentes tecnologias relativas às áreas de atuação, [...] capacidade de pesquisar, buscar informações, analisá-las e selecioná-las; a capacidade de aprender, criar, formular, ao invés do simples exercício de memorização (BRASIL, 2000, p.5).

É inegável o papel das tecnologias na inclusão, todavia a formação inicial e a prática também são fundamentais é o que aponta o artigo "Deficiência Visual e Educação Matemática: estudo dos artigos publicados nos anais dos Encontros Nacionais de Educação Matemática". Neste Cintra e Beirigo (2019) realizam uma pesquisa de cunho qualitativo com objetivo de apresentar os estudos que interpelam a deficiência visual difundidos em todos os anais dos Encontros Nacionais de Educação Matemática. A partir do levantamento Cintra e Beirigo (2019) concluem que:

[...] a inclusão dos alunos com NEE ganhou repercussão em eventos acadêmicos, a partir de 2004 e acreditamos que tal fato está associado às demandas dos avanços da legislação brasileira em direção a educação inclusiva. Vale destacar que dos 38 trabalhos encontrados, 28 se referem a atividades didáticas, ou seja, mais de $70 \%$ das pesquisas realizadas estão preocupadas em desenvolver metodologias de ensino adequadas e eficientes para a instrução da Matemática para aluno com deficiência visual.

Consonantes com o já exposto por outros autores Ferreira, Corrêa e Boron (2018) em "O ensino da matemática para portadores de deficiência visual" aprofundam a inclusão em seu aspecto para além da sala de aula, segundo as autoras devem existir transformações na sociedade com vista a proporcionar a todos as mesmas oportunidades. $\mathrm{O}$ trabalho comunga com os demais ao discorrer sobre a falta de preparo dos professores para receberem alunos com diferentes necessidades de aprendizagem segundo as autoras:

O ensino da Matemática a portadores de deficiência visual fica disperso e inconsistente se não se adotar meios de "visualização" de gráficos, equações e figuras geométricas, pois se precisa muito do apoio visual, para melhor ensinar e ser compreendido pelos alunos. Além disso, são apresentados vários recursos e materiais manipuláveis para o ensino da Matemática, que podem tornar as aulas mais agradáveis e interessantes, tanto para educandos portadores de deficiência visual como para os demais.

A utilização de diferentes recursos e materiais manipuláveis favorece a inclusão por propiciar a construção do conhecimento de forma autônoma pelo aluno com deficiência visual e promover a interação deste aluno com os demais estudantes (Marcelly, 2015).

\section{Considerações Finais}

O desenvolvimento do presente estudo possibilitou uma análise de como ocorre o ensino de matemática para alunos com deficiência visual, além de inferir sobre como os professores se sentem frente ao processo de inclusão destes alunos em salas de aulas regulares. Além disto analisamos os desafios e potencialidades na transposição da linguagem científica no ensino de matemática para alunos com deficiência visual.

Verificou-se uma carência na quantidade de trabalhos que discorrem sobre a deficiência visual e o ensino de matemática, esta perspectiva é constatada pelo estudo realizado por Cintra e Beirigo (2019).

Um aspecto relevante verificado na análise dos trabalhos e que deve ser apontado: os autores e professores reconhecem a necessidade do desenvolvimento de materiais didáticos pedagógicos que favoreçam a construção do conhecimento científico matemático além da premência de uma formação que contemple as diferentes necessidades de aprendizagem 
Assim podemos inferir que a falta de preparo dos professores, das escolas, e a carência de materiais pedagógicos adaptados como desafios a serem superados a fim de transpor a linguagem científica para as pessoas com diferentes necessidades de aprendizagem. O desenvolvimento de manuais específicos de como realizar de maneira satisfatória esta transposição é segundo Villani e Nascimento (2003) uma das possibilidades de favorecer a inclusão.

\section{Referências}

Moreira, I. D. C. (2002). Ciência e público: caminhos da divulgação científica no Brasil (No. 001.9 CIE).

De Paula Cintra, V., \& Beirigo, J. A. C. (2019). Deficiência Visual e Educação Matemática: estudo dos artigos publicados nos anais dos Encontros Nacionais de Educação Matemática. Ensino em Re-Vista, 1261-1285.

Da Luz Ferreira, A., Corrêa, E. M. M. M., \& da Silva Boron, F. C. O Ensino da Matemática para Portadores de Deficiência Visual.

Freire, P. (1996). Pedagogia da autonomia: saberes necessários à prática pedagógica. São Paulo: Paz e Terra.

Visual, D., \& Gil, M. (2000). Brasília: MEC. Secretaria de Educação a Distância.

Gil, A. C. (2002). Como elaborar projetos de pesquisa. São Paulo: Atlas, 1993. GIL, Antônio Carlos. Como elaborar projetos de pesquisa. São Paulo: Atlas.

Escolar, C. (2015). INEP 2013 disponível em http://www. qedu. org. br/escola/241374-eeespecial-professor-abnael-machado-de-lima-cene/sobre. Acesso em, 21(06).

Escolar, C. (2020). INEP 2013 disponível em http://www. qedu. org. br/escola/241374-eeespecial-professor-abnael-machado-de-lima-cene/sobre. Acesso em, 21(08).Manning, P. K. (1979). Metaphors of the field: Varieties of organizational discourse. Administrative Science Quarterly, 24(4), 660-671.Mantoan, M. T. E., \& Prieto, R. G. (2003). Inclusão escolar: o que é. Por quê, 12.

Moreira, M. A. (2004). Pesquisa básica em educação em ciências: uma visão pessoal. Revista Chilena de Educación Científica, $3(1), 10-17$.

Mamcasz-Viginheski, L. V., da Silva, S. D. C. R., \& Shimazaki, E. M. (2019). Ensino de conceitos matemáticos para estudante com deficiência visual em situação de inclusão. Educação Matemática Pesquisa: Revista do Programa de Estudos Pós-Graduados em Educação Matemática, 21(3).

Pasquarelli, R. D. C. C., \& Manrique, A. L. (2016). A inclusão de estudantes com deficiência visual no ensino e aprendizagem de estatística: medidas de tendência central The inclusion of students with visual impairments in the teaching and learning of statistics: measures of central tendency. Educação Matemática Pesquisa: Revista do Programa de Estudos Pós-Graduados em Educação Matemática, 18(1).

Silva, R. S. (2020) A defectologia e a Teoria Histórico Cultural no Ensino de Química para o Deficiente Visual: Um estado da arte em teses e dissertações (2000-2019).

Silva, R. S., \& Amaral, C. L. C. (2020). A educação inclusiva no ensino de ciências e matemática. Communitas, 4(7), 281-294.

Silva, R. S., \& Carmem Lúcia Costa Amaral. (2020). Percepção de professores de química face à educação de alunos com deficiência visual: dificuldades e desafios. South American Journal of Basic Education, Technical and Technological , 7(1), 108-129.

Shimazaki, E. M., da Silva, S. D. C. R., \& Viginheski, L. V. M. (2015). O ensino de matemática e a diversidade: o caso de uma estudante com deficiência visual. Interfaces da Educação, 6(18), 148-164.

Vigotski, L. S. (1997). Obras escogidas V: Fundamentos de defectología. Madrid: Visor, 5. 